\title{
Remarkable diversity of intron- 1 of the para voltage-gated sodium channel gene in an Anopheles gambiae/Anopheles coluzzii hybrid zone
}

Federica Santolamazza', Beniamino Caputo ${ }^{1}$, Davis C Nwakanma², Caterina Fanello ${ }^{3}$, Vincenzo Petrarca ${ }^{4}$, David J Conway ${ }^{2}$, David Weetman ${ }^{5}$, Joao Pinto ${ }^{6}$, Emiliano Mancini ${ }^{1,7^{*}}$ and Alessandra della Torre ${ }^{1}$

\begin{abstract}
Background: Genomic differentiation between Anopheles gambiae and Anopheles coluzzii - the major malaria vectors in sub-Saharan Africa - is localized into large "islands" toward the centromeres of chromosome-X and the two autosomes. Linkage disequilibrium between these genomic islands was first detected between species-specific polymorphisms within ribosomal DNA genes (IGS-rDNA) on the X-chromosome and a single variant at position 702 of intron 1 (Int-1 ${ }^{702}$ ) of the para Voltage-Gated Sodium Channel (VGSC) gene on chromosome arm $2 \mathrm{~L}$. Intron-1 sequence data from West and Central Africa revealed two clearly distinct and species-specific haplogroups, each characterized by very low polymorphism, which has been attributed to a selective sweep. The aim of this study was to analyse Int-1 sequence diversity in A. gambiae and A. coluzzii populations from the Far-West of their range, in order to assess whether this selective-sweep signature could persist in a zone of high interspecific hybridization.
\end{abstract}

Methods: A 531 bp region of VGSC Int-1 was sequenced in 21 A. coluzzii, 31 A. gambiae, and 12 hybrids from The Gambia and Guinea Bissau, located within the Far-West geographical region, and in 53 A. gambiae s.l. samples from the rest of the range.

Results: Far-West samples exhibit dramatic Int-1 polymorphism, far higher within each country than observed throughout the rest of the species range. Moreover, patterning of haplotypes within A. coluzzii confirms previous evidence of a macro-geographic subdivision into a West and a Central African genetic cluster, and reveals a possible genetic distinction of $A$. coluzzii populations from the Far-West.

Conclusions: The results suggest a relaxation of selective pressures acting across the VGSC gene region in the hybrid zone. Genetic differentiation in the Far-West could be attributable to a founder effect within A. coluzzii, with subsequent extensive gene flow with secondarily-colonizing A. gambiae, potentially yielding a novel insight on the dynamic processes impacting genetic divergence of these key malaria vectors.

Keywords: Mosquito, Malaria, Hybridization

\footnotetext{
* Correspondence: emiliano.mancini@uniroma1.it

'Dipartimento di Sanità Pubblica e Malattie Infettive, Istituto

Pasteur-Fondazione Cenci-Bolognetti, Università "Sapienza", Piazzale Aldo

Moro 5, 00185 Rome, Italy

${ }^{7}$ Dipartimento di Scienze, Università Roma Tre, Viale Marconi 446, 00146

Rome, Italy

Full list of author information is available at the end of the article
}

\section{Biomed Central}

(c) 2015 Santolamazza et al.; licensee Biomed Central. This is an Open Access article distributed under the terms of the Creative Commons Attribution License (http://creativecommons.org/licenses/by/4.0), which permits unrestricted use, distribution, and reproduction in any medium, provided the original work is properly credited. The Creative Commons Public Domain Dedication waiver (http://creativecommons.org/publicdomain/zero/1.0/) applies to the data made available in this article, unless otherwise stated. 


\section{Background}

Mosquito species belonging to the Afro-tropical Anopheles gambiae complex represent a valuable model for studies of ecological speciation ('speciation with gene flow') $[1,2]$. In fact, owing to the major role of some of these species in malaria transmission, their genetic divergence has been studied extensively for more than half century, revealing repeated events of 'ecotypic speciation' $[3,4]$. These studies have revealed the existence of morphologically indistinguishable, but chromosomally/ genetically distinct species with very different roles as malaria vectors (A. gambiae sensu stricto, Anopheles arabiensis, Anopheles quadriannulatus, Anopheles amharicus, Anopheles melas, Anopheles merus, Anopheles bwambae) [5,6]. In addition to these sibling species, which are isolated by both pre-and post-mating mechanisms, more recent studies have highlighted a further subdivision within the nominal species $A$. gambiae s.s., the most synanthropic and efficient malaria vector of the complex $[7,8]$. These studies have revealed the existence of two taxonomic units initially named the ' $\mathrm{M}$ ' and ' $\mathrm{S}$ ' molecular forms and now formally raised to species as Anopheles coluzzii and A. gambiae s.s. (hereafter referred as A. gambiae) [9], respectively. The two species are isolated only by (partially understood) pre-mating mechanisms and show limited genomic differentiation, which is localized most prominently in low-recombination pericentromeric regions of chromosome- $\mathrm{X}$ and of the two autosomes [10-13]. These regions have been postulated to represent 'genomic islands of speciation', expanding in size by selection across linked loci connected to reproductive isolation, as predicted by ecological speciation with gene flow models [1,2]. However, their large size is probably enhanced by locally reduced recombination $[10,14,15]$, leading to suggestions that high centromeric differentiation is primarily a result of recurrent background selection and hitchhiking unrelated to speciation $[2,16]$. Interestingly, the species-specific linkage disequilibrium among the three physically-unlinked centromeric regions is widespread [13], but has been lost at the western extreme of their range (i.e. the 'Far-West', from The Gambia to Guinea Bissau). In this putative secondary contact zone between the two species, high frequencies of hybrids are found and pronounced inter-specific differentiation is maintained only on the chromosome-X centromere [17-21].

The first evidence of genetic linkage between the chromosome-X and chromosome- 2 genomic island regions came from pre-genomic studies showing that the SNPs within the ribosomal intergenic spacer region (IGS - rDNA), which define the two species [7], are in strong linkage disequilibrium (LD) with a SNP at position 702 of Intron-1 (hereafter named Int- ${ }^{702}$ ) of the para Voltage-Gated Sodium Channel (VGSC) gene [22,23]. The
VGSC gene has been studied extensively because of the presence of two mutations in the exon immediately downstream of Int-1, causing a change from Leucine to Serine (L1014S) or to Phenylalanine (L1014F). Each mutation can confer knock-down resistance $(k d r)$ to DDT and pyrethroid insecticides [22,23]. In West and Central Africa the Int- $1^{702}$ SNP, which exhibits diagnostic nucleotides for each species (i.e. A. coluzzii $=$ Int- $1^{\mathrm{C}}$; A. gambiae $=$ Int $-1^{\mathrm{T}}$ ), defines two clearly distinct and species-specific haplotype groups each characterized by very low polymorphism. This led to the hypothesis of a selective sweep, pre-dating $k d r$ mutations, centred on favourable variants in nearby genes that might contribute to the segregation of the two species [23].

The aim of this study was to investigate sequence diversity of the Int-1 region of the VGSC in A. gambiae and $A$. coluzzii populations from the Far-West to assess whether the selective-sweep observed in the rest of the species range is maintained in the face of high interspecific gene flow.

\section{Methods}

\section{Anopheles gambiae sensu lato sample identification and} kdr genotyping

Anopheles gambiae s.l. specimens were identified as A. arabiensis, A. quadriannulatus, A. melas or A. merus using the method of Scott et al. [24], and as A. coluzzii or A. gambiae s.s. by using both PCR-RFLP of the intergenic spacer (IGS) rDNA region [25] and the SINEPCR [26] methods. The latter method is based on an $A$. coluzzii-specific and irreversible single-locus insertion of a SINE200 retrotransposon in the X-chromosome centromeric region, about $1.5 \mathrm{Mb}$ from IGS region containing A. coluzzii vs A. gambiae species-specific SNPs. The $k d r 1014$ locus was genotyped by either allelespecific-PCR (AS-PCR) or Hot Oligonucleotide Ligation Assay (HOLA) methods [27-29] in A. coluzzii and A. gambiae. Genotypes of $k d r 1014$ position in A arabiensis, A. quadriannulatus, A. melas and A. merus were obtained by direct sequencing of a $531 \mathrm{bp}$ fragment [27].

\section{Sequencing of Int-1 of VGSC gene}

Amplification of a 531 bp region of Int-1 of the VGSC gene (AGAP004707 in genome build AgamP4.1) was carried out using the "Ganest" primer (5'- CAT ACA TTG CTT AAA GCT CTA ATT ATC -3'), located upstream at positions $388-414$ in the Int-1 region, coupled with the "Montrev" primer (5'- CAC AAG GCA CAC GAT ACG -3'), located downstream at positions 9951013 at the end of intron-2 (nucleotide positions as in [22]). The PCR mixture contained: 10× PCR Buffer (Bioline), $2 \mathrm{mM} \mathrm{MgCl} 2,200 \mu \mathrm{M}$ dNTPs equimolar mix, 1 $\mathrm{U}$ Taq DNA polymerase (Bioline) and $0.25 \mu \mathrm{M}$ of each primer, in a total volume of $25 \mu \mathrm{l}$. Cycling conditions 
were as follows: $94^{\circ} \mathrm{C}$ for $5 \mathrm{~min}$., 35 cycles each with $94^{\circ} \mathrm{C}$ for $30 \mathrm{sec} . / 50^{\circ} \mathrm{C}$ for $35 \mathrm{sec} . / 72^{\circ} \mathrm{C}$ for $1 \mathrm{~min}$., followed by a final extension step of $10 \mathrm{~min}$ at $72^{\circ} \mathrm{C}$. Direct sequencing was performed according to [22] and [23].

Chromatograms were edited using the Staden Package ver. 2003.1.6 [30] and sequences were aligned using MAFFT ver. 5 [31]. Haplotype inference was performed with the PHASE algorithm [32] as implemented in DnaSP v5.10.01 [33]. Haplotype sequences reported in Gentile et al. [23] were also included for comparison. Sequences were deposited in Genbank under accession n ${ }^{\circ}$ KP300645KP300752.

\section{Data analysis}

DnaSP v. 5.10.01 [33] was used to produce estimates of Int-1 polymorphism and perform Tajima's $D$ and $\mathrm{Fu}$ and Li's $D^{*}$ and $F^{*}[34,35]$ neutrality tests. Genealogical relationships among Int-1 haplotypes were reconstructed by computing parsimony networks using TCS 1.21 [36]. Haplotypes names were retained for those previously reported in [23], while novel M1- and S1-related haplotypes were either named with consecutive numbers or with specific codes when exclusive of the Far-West region (i.e. GU for Guinea Bissau, GA for The Gambia, and GUGA for both countries). Frequency and distribution of Int-1 haplotypes in the Far West, West and Central African geographic regions in A. coluzzii, A. gambiae and putative hybrids (by merging original and previous data from [23]) were also computed. Inter- and intra-specific Fst estimates of genetic differentiation were calculated using Arlequin 3.11 [37].

\section{Results}

A 531 bp region of the VGSC gene Int-1 was sequenced in 21 A. coluzzii, 31 A. gambiae, 12 A. gambiae x A. coluzzii hybrids from the Far-West region. These sequences were aligned with those obtained by Gentile et al. [23] and with 53 additional original sequences from $A$. coluzzii and $A$. gambiae populations from the rest of the distribution range, as well as from other species of the complex (Additional file 1: Table S1), resulting in a total of 490 sequences. Note that $A$. coluzzii and A. gambiae were identified by both the IGS marker and SINE insertion, which provided completely consistent identification in all samples, with the notable exceptions of those from The Gambia (55\% inconsistent) and Guinea Bissau (51\% inconsistent). Inconsistently identified individuals were classified based on the SINE marker, as this is not biased toward the hybrid genotype by intra-chromosome recombination known to occur in the IGS multicopy rDNA region [38]. Specimens were genotyped for $k d r-$ mutations: all carried the wild type/insecticide $k d s$ susceptible allele of VGSC gene (i.e. TTA) with the exception of two out of eight individuals from Rwanda carrying the TCA allele (hereafter L1014S) in homozygosis and three carrying the TTT allele (hereafter L1014F) in heterozygosis (Additional file 2: Table S2).

Variable sites among haplotypes, and their relationships among different members of the A. gambiae complex, are shown in Figure 1 and in Figure 2, respectively. All species are well-separated from each other and from A. coluzzii and A. gambiae (except for one A. arabiensis individual from Senegal sharing the S1-haplotype with A. gambiae). Interestingly, A. gambiae carries a C to T mutation at site 702 (hereafter Int-1 ${ }^{702}$, in red in Figure 1) separating it from all other species. This mutation was already shown by Gentile et al. [23] to separate "S-molecular form-S1 (Int- $1^{\mathrm{T}}$ )" from "M-form-M1 (Int-1 ${ }^{\mathrm{C}}$ )" haplotypes and closely-related locale-specific variants. In the Gambian and Guinean samples, however, $A$. coluzzii-specific Int- ${ }^{\mathrm{C}}$ haplotypes are also found in A. gambiae, but no A. gambiae-specific Int- $1^{\mathrm{T}}$ haplotypes are found in $A$. coluzzii (Figures 2b and 2c; Additional file 2: Table S2). This result does not change if the species are identified using the IGS marker rather than by SINE-PCR, as presented so far.

Overall, Int-1 haplotypes are not uniformly distributed across the ranges of A. gambiae and A. coluzzii (Figure 3). Populations from the Far-West region (i.e. Senegal, The Gambia, Guinea Bissau, Guinea Conakry) differ from all others by the exclusive presence of the M3 haplotype (freq $=18 \%$ in A. gambiae, $32 \%$ in A. coluzzii and 16\% in hybrids). Moreover, populations from The Gambia and Guinea Bissau possess 15 exclusive haplotypes (freq $=20 \%$ in both A. gambiae and A. coluzzii, $29 \%$ in hybrids), highlighted in yellow in Figure 2. M1 is the most frequent haplotype in both West and in Central African A. coluzzii populations, while the M5 haplotype reaches frequencies up to $30 \%$ in West African samples and is absent in Central ones. Haplotype S1 is almost the sole haplotype found in western A. gambiae populations (Mali, Ivory Coast, Burkina Faso and Nigeria; freq $=99 \%$ ), whereas S1-related haplotypes are much more frequent in central ones (i.e. Cameroon and Angola; freq. $=22 \%$ ). Finally, both $\mathrm{S} 1$ and the $A$. coluzzii-specific M1 haplotype are found at comparable frequencies (nearly 50\% each) in A. gambiae from Rwanda (Additional file 2: Table S2).

Differentiation between A. coluzzii and A. gambiae in Central Africa $(F s t=0.69)$ is comparable to that observed between each species and $A$. arabiensis ( $F s t=0.70$ and 0.77, respectively), but decreases westwards (Fst in West-Africa $=0.51$ and in Far-West region $=0.16$ ) (Figure 4). Intraspecific Fst values are slightly lower between West and Central populations of each species $(F s t=0.04$ and 0.14) than between Far-West samples and West and Central ones (Fst ranging from 0.19 to 0.31 ) (Figure 4).

Table 1 shows genetic diversity and summary statistics for A. coluzzii and A. gambiae samples from Far-West, West, Central Africa, as well as from Rwanda, including 


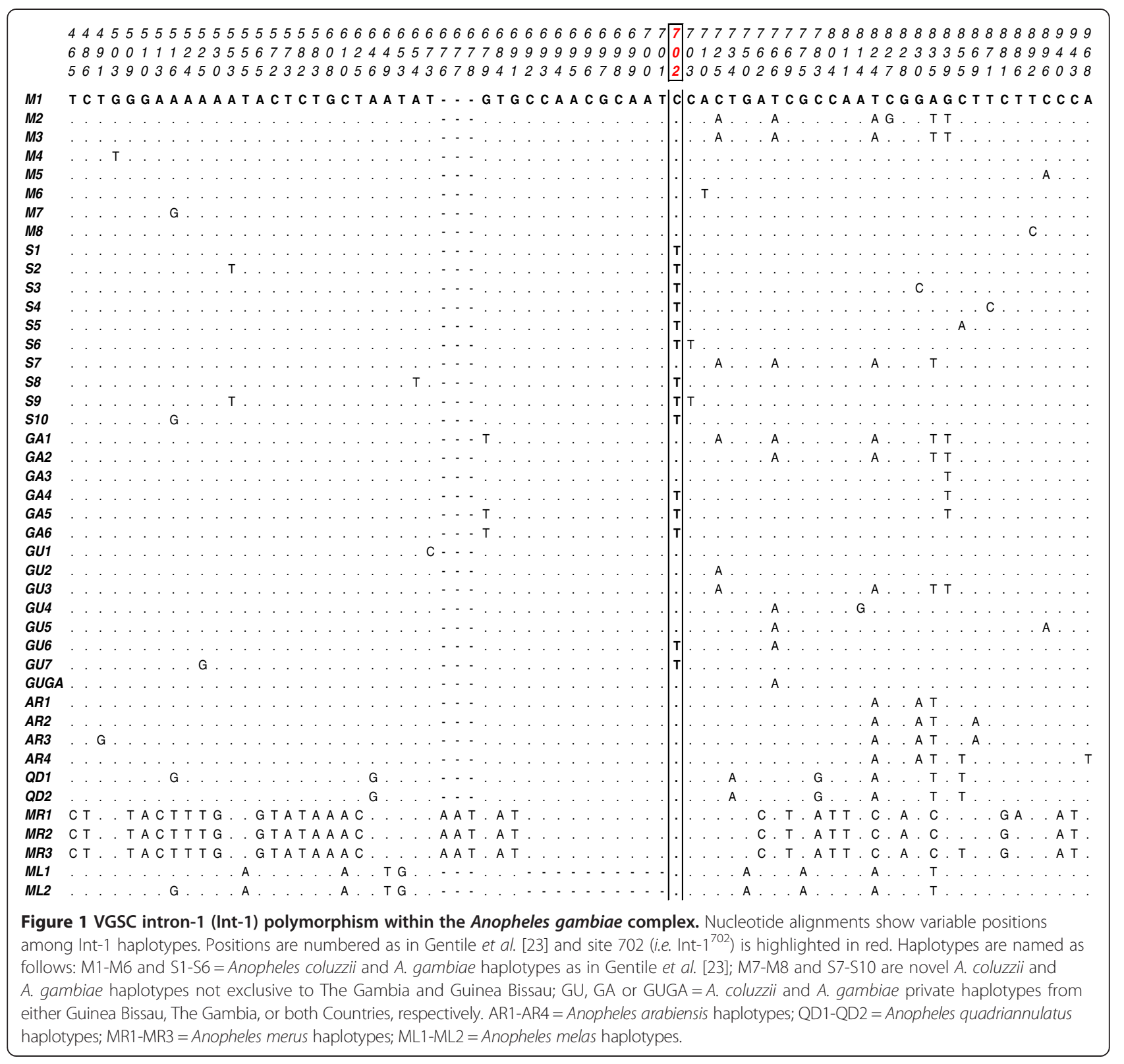

original data and those from West/Central Africa reported by Gentile et al. [23]. In general, nucleotide/ haplotype diversity is low in samples from West and Central Africa $(\pi=0.02-0.21)$, but increases in the FarWest region $(\pi=0.47-0.49)$. Statistics applied to detect departures from neutrality reveal a weak trend toward positive values in A. gambiae and A. coluzzii (and hybrids) from "Far-West", whereas negative values were scored in both species from West and Central Africa (significant in A. gambiae: $D^{*}=-2.583, F^{*}=-2.686$, and A. coluzzi: $\left.D^{*}=-2.848, F^{*}=-2.922\right)$ as well as in A. gambiae from Rwanda, suggesting the influence of different selective pressures and/or demographic histories in populations from different geographic areas.

\section{Discussion}

Data on Int-1 of the VGSC gene highlight dramatic genetic differences between $A$. coluzzii and A. gambiae populations from West and Central Africa and those from the western extreme of the species range, where inter-specific geneflow is elevated and inter-specific differentiation reduced.

In West and Central Africa, Int-1 genetic differentiation between $A$. coluzzii and A. gambiae is high and comparable to that observed in their sibling A arabiensis (Figure 4). Across this wide geographical region, as previously shown [23], the two species exhibit low nucleotide/haplotype diversity and are strongly segregated based on two main Int-1 haplotypes (A. coluzzii-M1 and $A$. gambiae-S1, separated by a single C-T mutational 
a)

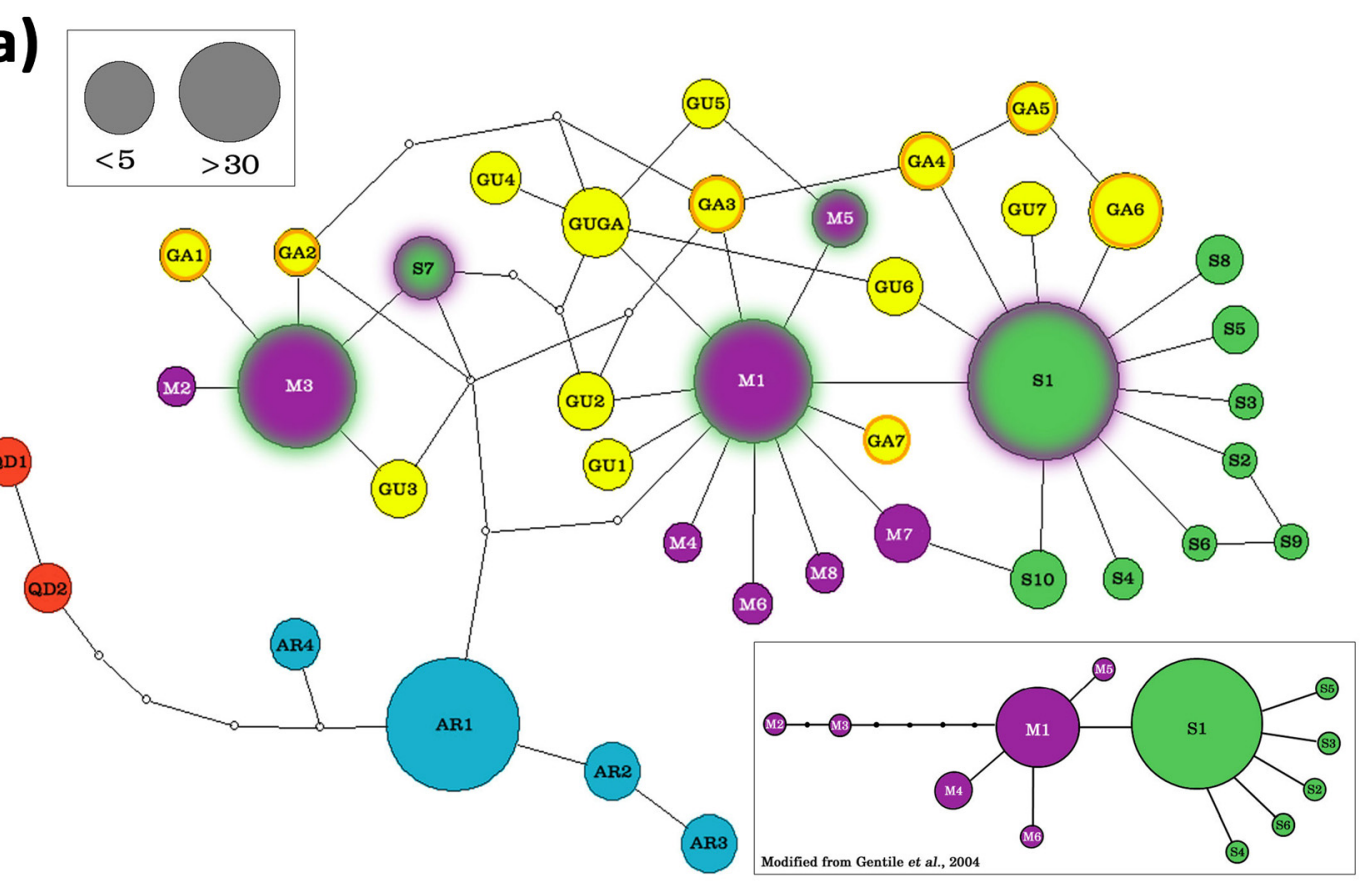

b)

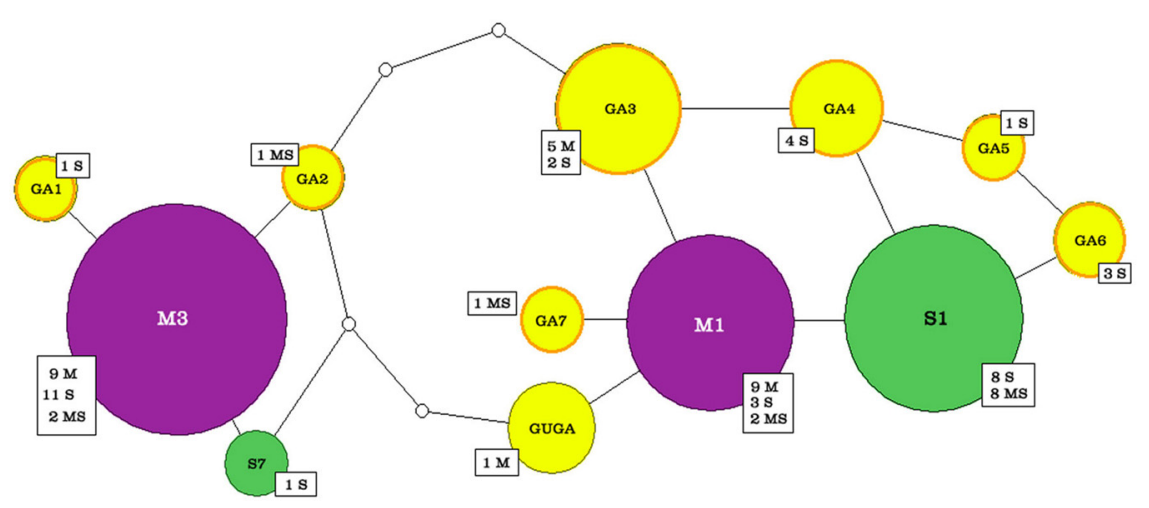

c)

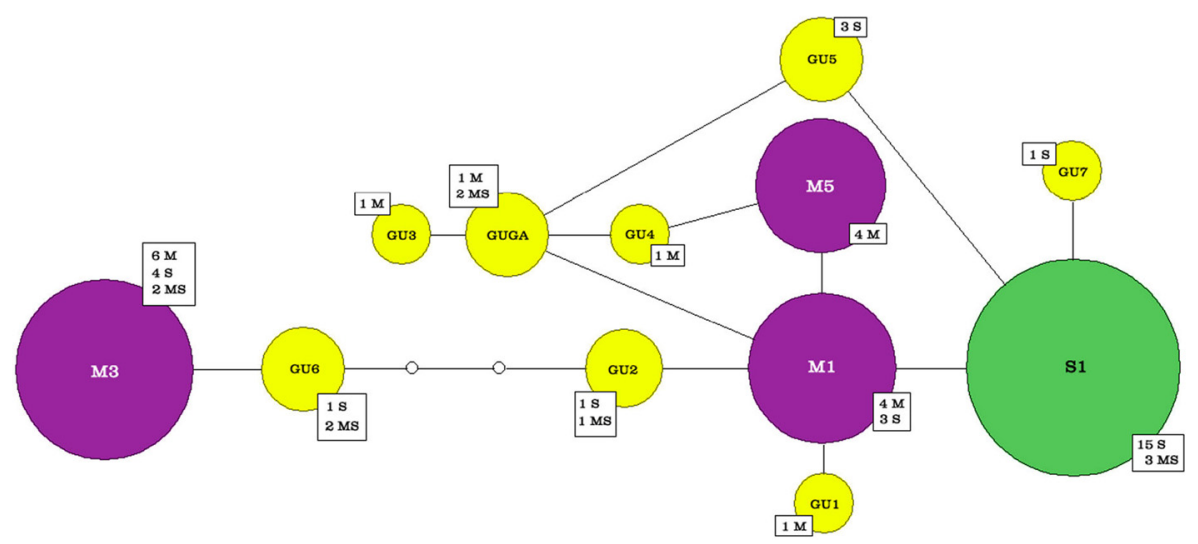

Figure $\mathbf{2}$ (See legend on next page.) 
(See figure on previous page.)

Figure 2 Parsimony-based networks of genealogical relationships among VGSC Intron-1 haplotypes in the Anopheles gambiae complex. a) Network built with original data collected from all studied species of the A. gambiae complex, with the exception of Anopheles melas and Anopheles merus, whose haplotypes exceeded the 95\% threshold of TCS connection limit. Haplotypes are represented by pies whose sizes are proportional to frequencies in the sample and coloured as follows: blue =Anopheles arabiensis (AR), red = Anopheles quadriannulatus (QD), violet $=$ Anopheles coluzzii, green = Anopheles gambiae (haplotypes are named and numbered according to Gentile et al. [23]), yellow = private haplotypes from either Guinea Bissau (GU), The Gambia (GA), or from both Countries (GUGA); sequential codes are used to name A. coluzzii (i.e. M7, M8) and A. gambiae (S7-S10) novel haplotypes not exclusive to The Gambia and Guinea Bissau; M1, M3, M5, S1 and S7, which are not completely segregated between the two species in the Far-West and/or Rwanda, are shaded. Below: VGSC Int-1 network for M (A. coluzzii) and S (A. gambiae) molecular forms as in Gentile et al. [23]; b) and c) networks only including A. coluzzii, A. gambiae and hybrids haplotypes from The Gambia and Guinea Bissau, respectively. White squares report numbers of alleles for A. coluzzii (M), A. gambiae (S) and hybrids (MS) (identified based on SINE-PCR [26]) included in each haplotype.

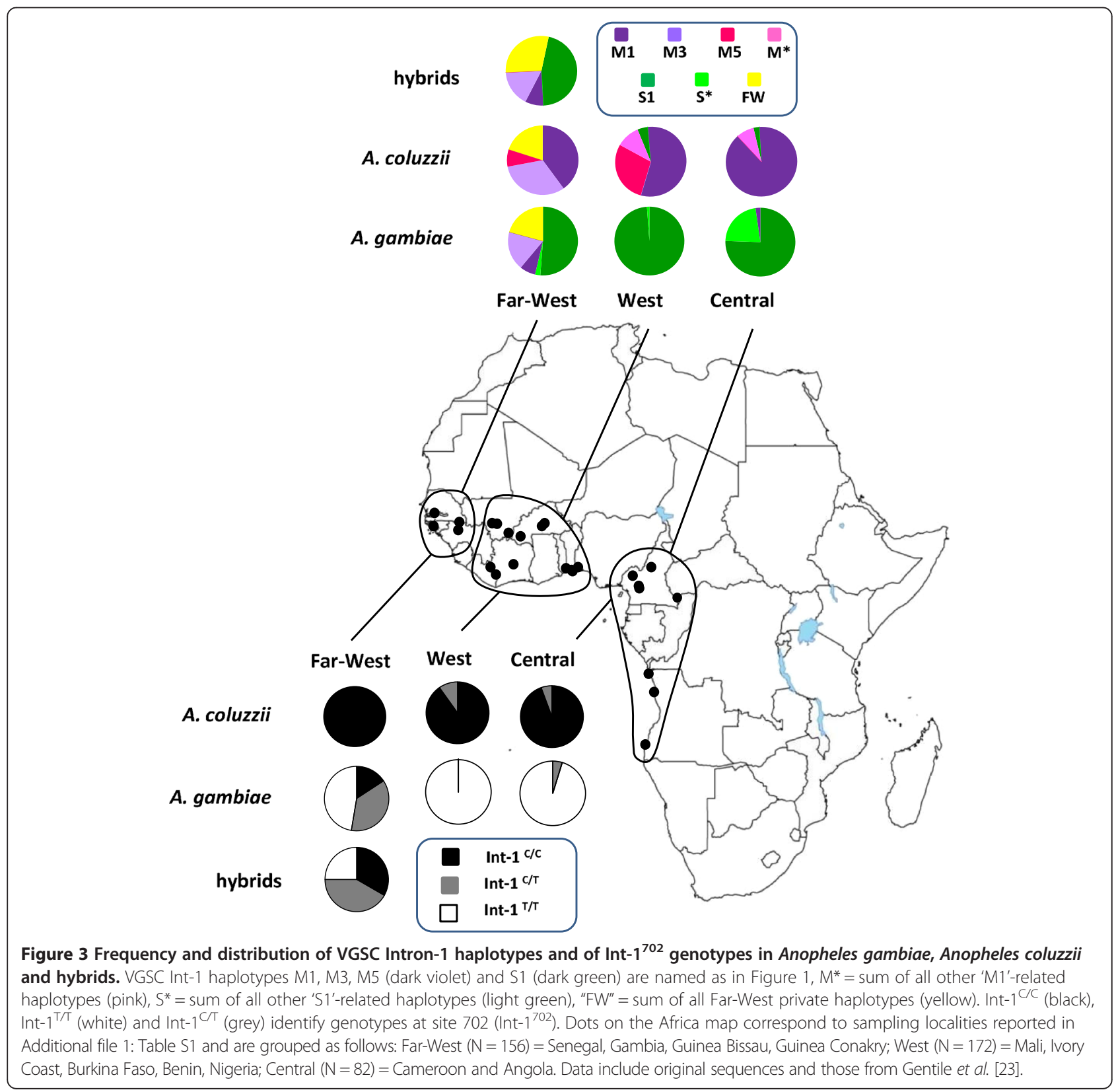




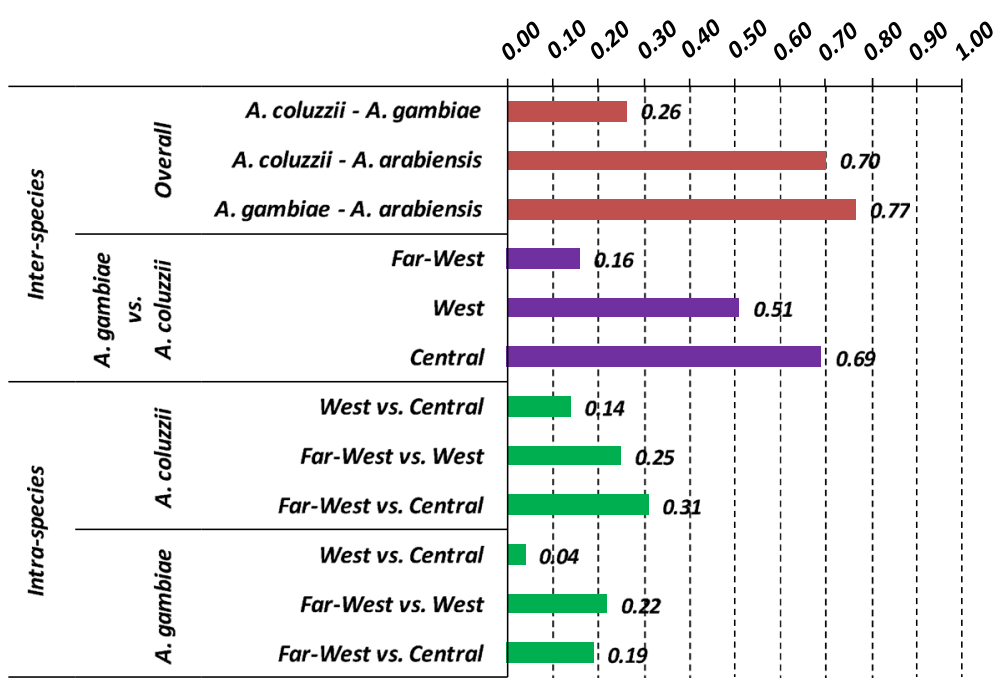

Figure 4 Inter- and intra-specific Fst values based on VGSC Intron-1 sequence data. Above: inter-specific Fst values among Anopheles gambiae, Anopheles coluzzii and Anopheles arabiensis (red) and between A. gambiae and A. coluzzii in each geographic region (violet). Below: intra-specific Fst values among populations from different geographic regions within A. coluzzii and A. gambiae (green). Fst are all significant $(p<0.05)$. Data include original sequences and those from Gentile et al. [23].

step at site Int- ${ }^{702}$ ) and species-specific rare variants stemming from these. To explain this low polymorphism, a selective sweep centered on a favourable variant in a nearby gene was suggested [22,23]. Since the VGSC gene includes mutations conferring $k d r$ resistance to insecticides, a possible hypothesis is that Int-1 haplotypes are in linkage with these strongly selected alleles. Introgression of $k d r$ alleles and adjacent genomic regions has repeatedly been reported from different geographic areas $[19,39]$. Indeed, whole genome sequence data have recently shown that over $3 \mathrm{Mb}$ has introgressed from A. gambiae to A. coluzzii in Ghana along with the $k d r$ L1014F mutation [40]. It is possible that selection on $k d r$-associated Int- 1 haplotypes may have had a role in reducing diversity, as $k d r$-resistance has been reported in some of the A. gambiae analysed populations particularly from West Africa [41,42] [but not in the Far-West region, Pinto et al., unpublished observations; see discussion below]. However, the close physical proximity of $k d r$ and Int-1 would make recombination between resistant and susceptible $k d r$ alleles and their linked Int-1 polymorphisms highly unlikely over a short timescale. Moreover, in A. coluzzii, as the samples analyzed came from populations where $k d r$-alleles were either absent or present at moderate to low frequencies (i.e. populations from Benin, 40.0\%, Nigeria, $19.5 \%$ and Cameroon, $6.3 \%$ [42]), and are

Table 1 Intron-1 of VGSC gene polymorphism and summary statistics in Anopheles coluzzii and A. gambiae samples

\begin{tabular}{|c|c|c|c|c|c|c|c|c|c|c|}
\hline & African region & $\mathbf{n}$ & $\mathrm{H}$ & Hd & $\mathrm{S}$ & $\pi(\%)$ & $\theta(\%)$ & Tajima D & Fu \& Li's $D$ & Fu \& Li's $F$ \\
\hline \multicolumn{11}{|l|}{ A. coluzzii } \\
\hline & Far-West & 50 & 8 & 0.73 & 8 & 0.49 & 0.34 & 1.217 & -0.127 & 0.361 \\
\hline & West & 92 & 7 & 0.68 & 11 & 0.21 & 0.41 & -1.303 & 0.120 & -0.450 \\
\hline & Central & 38 & 4 & 0.15 & 3 & 0.03 & 0.14 & -1.720 & $-2.848^{*}$ & $-2.922^{*}$ \\
\hline \multicolumn{11}{|c|}{ A. gambiae } \\
\hline & Far-West & 82 & 14 & 0.70 & 9 & 0.47 & 0.34 & 0.926 & -0.123 & 0.277 \\
\hline & West & 78 & 3 & 0.12 & 2 & 0.02 & 0.08 & -1.121 & -1.011 & -1.218 \\
\hline & Central & 46 & 8 & 0.51 & 7 & 0.12 & 0.30 & -1.657 & $-2.583^{*}$ & $-2.686^{*}$ \\
\hline & East & 16 & 4 & 0.65 & 6 & 0.22 & 0.34 & -1.230 & -2.025 & -2.076 \\
\hline \multicolumn{11}{|l|}{ Hybrids } \\
\hline & Far-West & 24 & 8 & 0.77 & 7 & 0.52 & 0.36 & 1.472 & 0.629 & 1.016 \\
\hline
\end{tabular}

Data include original sequences and those from Gentile et al. [23]. Far-West = Senegal, Gambia, Guinea Bissau, Guinea Conakry; West = Mali, Ivory Coast, Burkina Faso, Benin, Nigeria; Central $=$ Cameroon and Angola; East $=$ Rwanda. $n=n^{\circ}$ alleles; $H=n^{\circ}$ haplotypes; $S=n^{\circ}$ segregating sites; $\mathrm{Hd}=$ haplotype diversity; $\pi=$ nucleotide diversity; $\theta=$ Watterson estimate of theta; for Tajima $D$, Fu \& Li's $D$ and $F$ values, $p$ computed using confidence levels provided by the coalescent (DNAsp 5.0) * $<0.05$. 
thus likely to have introgressed very recently $[19,22,39]$. Recent genomic studies have given additional hints for understanding the reduction in genetic variation at Int-1 and its linkage disequilibrium with markers on the physically unlinked X-centromeric region defining the two species. In fact, as already mentioned, the VGSC gene is located within the chromosome-2 "genomic island" of highest divergence between A. coluzzii and A. gambiae. Under the "speciation island" [10] scenario, it can be hypothesized that a hitchhiking effect on Int-1 has occurred due to diversifying selection on a chromosome-2 "island" gene participating in the building-up of premating barriers, or conferring differential ecological adaptation and niche segregation between $A$. gambiae and $A$. coluzzii. Alternatively, under the "incidental island" scenario $[2,13,16]$, substitutions at Int- $1^{702}$ may have become fixed after species splitting and accumulated little genetic differentiation due to reduced recombination in the chromosome- 2 centromeric region. Interestingly, however, the association between chromosome-X and -2 "islands" is neither observed in Rwanda (Additional file 2: Table S2) nor in Tanzania [41]. In these East African sites, both M1 and S1 haplotypes were found segregating in $A$. gambiae populations. Thus, if Int- $1^{\mathrm{C}}$ represents the ancestral allele in the A. gambiae complex (Figure 1), then Int-1 ${ }^{\mathrm{C} / \mathrm{T}}$ may be considered an ancestral polymorphism retained in A. gambiae populations from East Africa (where A. coluzzii is absent), which became fixed in westward sympatric areas after the splitting of the two species.

In the Far-West region, a strong reduction of interspecific genetic divergence between $A$. gambiae and A. coluzzii is found - as indicated by the lower Fst observed in this region (0.14) as opposed to the rest of the range (0.51-0.69) (Figure 4) - and a preferential introgression of M1-related Int-1 haplotypes ("typical" of A. coluzzii) into A. gambiae is observed. These data are consistent with previous studies showing weak association between chromosome- $\mathrm{X}$ and -2 centromeric regions and occurrence of asymmetric introgression from A. coluzzii into A. gambiae in the westernmost extreme of their range [17-21]. Furthermore, 15 exclusive FarWest haplotypes were inferred through PHASE [32] and found interspersed and connected to M1 and S1 geographically widespread variants and to the Far-Westspecific M3 (Figure 2). The presence of such private haplotypes might indicate that selective pressures on the chromosome- 2 centromere observed in Central African populations (Table 1) are relaxed in the Far-West. Note that, although recombination along the centromeric 500-bp Int-1 fragment analyzed would normally be considered minimal, some reduction in accuracy might occur when reconstructing haplotypes using the PHASE algorithm in the Far-West region, where LD along the
$2 \mathrm{~L}$-centromere is known to be lower than in the rest of the species range [19]. However, the PHASE results are supported by summary statistics (Table 1) also indicating extreme Int-1 diversity and recent introgression events in Far-West populations of both species.

There are contrasting possible explanations for the remarkable Int-1 polymorphism observed in the Far-West region. Under the 'speciation island' hypothesis [10] relaxation of diversifying selection on a key isolating trait on chromosome- 2 centromeric "island" (of which Int-1 is a part) may have contributed to weaken pre-mating barriers between A. gambiae and A. coluzzii and promote a higher rate of gene-flow in the Far-West region. This hypothesis, however, is in contrast with data from other West and Central African areas, where introgression from A. gambiae to A. coluzzii of a $k d r$-related genomic portion in linkage with Int-1 does not produce an increase in hybridization rates [21]. Alternatively, the genomic region linked to Int-1 may be not related to speciation $[2,16]$ and the observed pattern in the FarWest region could be attributed to a relaxation of purifying selection operating separately within each species on adaptive genetic traits not directly (or only weakly) involved in reproductive isolation. Hence, following this hypothesis, increased Int-1 polymorphism in the "FarWest" region might be the consequence of an increased recombination rate within the $2 \mathrm{~L}$-centromeric "island" (and Int-1) following disruption of linked (background) selection. Resolution of these competing hypotheses requires assessment of the role of hybridization on the extent of linkage disruption throughout the 2 L- "island" and understanding of whether and how this might affect association with traits critical to speciation.

Finally, the frequency and distribution of Int-1 haplotypes within A. coluzzii across its range provides some hints on further intra-specific geographical patterns (Figure 3). In fact, populations from the West and FarWest regions are characterized by the exclusive presence of haplotype M5, not observed in those from Central Africa. This is consistent with results obtained by other nuclear markers (e.g. microsatellites) showing a macrogeographic subdivision into two distinct West and Central African genetic clusters, corresponding to the forestsavannah biome transition, which may have acted as an ecological barrier to gene flow $[26,43,44]$. Moreover, the high frequency of the Far-West exclusive M3-haplotype (separated from the major and widespread M1-haplotype by 5 mutational steps) allows speculation that a founder effect (followed by either selection or drift) affected A. coluzzii populations colonizing this region in the past. This last point merits further investigation through a multi-locus approach at a wider genome scale to shed light on the genetic characteristics of source populations originating the A. gambiae/A. coluzzii hybrid zone. 


\section{Additional files}

Additional file 1: Table S1. Anopheles gambiae complex sample for Intron-1 of VGSC gene sequencing data. GA = A. gambiae; $\mathrm{CO}=$ Anopheles coluzzii; $\mathrm{H}=$ putative $A$. coluzzii $\times$ A.gambiae hybrids; $A R=$ Anopheles arabiensis; $\mathrm{QD}=$ Anopheles quadriannulatus; $\mathrm{ML}=$ Anopheles melas; $\mathrm{MR}=$ Anopheles merus. CO, GA and H are defined based on SINE-PCR (Santolamazza et al., 2008).

Additional file 2: Table S2. Intron-1 genotypes in Anopheles coluzzii and Anopheles gambiae (and putative hybrids) sampled in this study. $\mathrm{N}=$ number of genotyped individuals; Int- $1^{702}=$ intron-1 genotype at position 702 (following Gentile et al., 2004); kdr=knock-down mutation at residue $1014:^{\S} \Pi \mathrm{A}=1014 \mathrm{~L}$ (wild type); $\Pi \Pi=1014 \mathrm{~F}$ (kdr-West); $\mathrm{TCA}=1014 \mathrm{~S}$ ( $k d r$-East).

\section{Competing interests}

The authors declare that they have no competing interests.

\section{Authors' contributions}

AdT, VP, DJC, FS conceived the study and participated in its design. FS, DCN carried out the molecular analyses. EM, BC, CF, JP performed the statistical analysis. EM, FS, DW, JP, AdT drafted the manuscript. All authors read and approved the final manuscript.

\section{Acknowledgements}

We are grateful to all scientists and entomology teams who contributed to mosquito samples, with particular reference to L. Iyikirenga (National Malaria Control Programme-TRAC Plus, Ministry of Health, Kigali, Rwanda), T.G.T Jaenson (Medical Entomology Unit, Department of Systematic Biology, Uppsala University, Uppsala, Sweden) and K. Palsson (KTH Royal Institute of Technology, Stockholm, Sweden). This work was supported by European Union's INFRAVEC project (grant agreement no. 228421 under FP7 program) to AdT and JP, Ricerca Scientifica 2012 grant by Università di Roma SAPIENZA to AdT and MIUR-FIRB "Futuro in Ricerca 2010" grant to BC (Grant N RBFR106NTE) and by CIRM ISS - Italian Malaria Network.

\section{Author details}

${ }^{1}$ Dipartimento di Sanità Pubblica e Malattie Infettive, Istituto Pasteur-Fondazione Cenci-Bolognetti, Università "Sapienza", Piazzale Aldo Moro 5, 00185 Rome, Italy. ${ }^{2}$ Medical Research Council Unit, Fajara, P.O. Box 273, Banjul, The Gambia. ${ }^{3}$ Centre for Tropical Medicine, Nuffield Department of Medicine, University of Oxford, Oxford, UK. ${ }^{4}$ Dipartimento di Biologia e Biotecnologie, Istituto Pasteur-Fondazione Cenci-Bolognetti, Università "Sapienza", Piazzale Aldo Moro 5, 00185 Rome, Italy. ${ }^{5}$ Vector Biology Department, Liverpool School of Tropical Medicine, Liverpool, UK. ${ }^{6}$ Centro de Malária e outras Doenças Tropicais, Instituto de Higiene e Medicina Tropical, Universidade Nova de Lisboa, Lisbon, Portugal. 'Dipartimento di Scienze, Università Roma Tre, Viale Marconi 446, 00146 Rome, Italy.

\section{Received: 6 August 2014 Accepted: 20 December 2014}

\section{Published online: 21 January 2015}

\section{References}

1. Feder JL, Egan SP, Nosil P. The genomics of speciation-with-gene-flow. Trends Genet. 2012;28:342-50.

2. Turner TL, Hahn MW. Genomic islands of speciation or genomic islands and speciation? Mol Ecol. 2010;19:848-50.

3. Coluzzi M. Spatial distribution of chromosomal inversions and speciation in anopheline mosquitoes. In: Barigozzi C, editor. Mechanisms of Speciation New York: Alan R. Liss Inc; 1982. p. 143-53.

4. Manoukis NC, Powell JR, Touré MB, Sacko A, Edillo FE, Coulibaly MB, et al. A test of the chromosomal theory of ecotypic speciation in Anopheles gambiae. Proc Natl Acad Sci USA. 2008;105:2940-5.

5. Coluzzi M, Sabatini A, della Torre A, Di Deco MA, Petrarca V. A polytene chromosome analysis of the Anopheles gambiae species complex. Science. 2002:298:1415-8.

6. Ayala FJ, Coluzzi M. Chromosome speciation: humans, Drosophila, and mosquitoes. Proc Natl Acad Sci USA. 2005;102:6535-42.

7. della Torre A, Fanello C, Akogbeto M, Dossou-yovo J, Favia G, Petrarca V, et al. Molecular evidence of incipient speciation within Anopheles gambiae s.s. in West Africa. Insect Mol Biol. 2001;10:9-18.
8. Della Torre A, Costantini C, Besansky NJ, Caccone A, Petrarca V, Powell JR, et al. Speciation within Anopheles gambiae: the glass is half full. Science. 2002;298:115-7.

9. Coetzee M, Hunt RH, Wilkerson R, della Torre A, Coulibaly MB, Besansky NJ. Anopheles coluzzii and Anopheles amharicus, new members of the Anopheles gambiae complex. Zootaxa. 2013;3619:246-74.

10. Turner TL, Hahn MW, Nuzhdin SV. Genomic islands of speciation in Anopheles gambiae. PLoS Biol. 2005;3:e285

11. Lawniczak MK, Emrich SJ, Holloway AK, Regier AP, Olson M, White B, et al. Widespread divergence between incipient Anopheles gambiae species revealed by whole genome sequences. Science. 2010;330:512-4.

12. Neafsey DE, Lawniczak MK, Park DJ, Redmond SN, Coulibaly MB, Traoré SF, et al. SNP genotyping defines complex gene-flow boundaries among African malaria vector mosquitoes. Science. 2010;330:514-7.

13. White BJ, Cheng C, Simard F, Costantini C, Besansky NJ. Genetic association of physically unlinked islands of genomic divergence in incipient species of Anopheles gambiae. Mol Ecol. 2010;19:925-39.

14. Via S, West J. The genetic mosaic suggests a new role for hitchhiking in ecological speciation. Mol Ecol. 2008;17:4334-45.

15. Feder $J \mathrm{~L}$, Nosil P. The efficacy of divergence hitchhiking in generating genomic islands during ecological speciation. Evolution. 2010;64:1729-47.

16. Cruickshank TE, Hahn MW. Re-analysis suggests that genomic islands of speciation are due to reduced diversity, not reduced gene flow. Mol Ecol. 2014;23:3133-57.

17. Caputo B, Santolamazza F, Vicente JL, Nwakanma DC, Jawara M, Palsson K, et al. The "far-west" of Anopheles gambiae molecular forms. PLoS One. 2011;6:e16415.

18. Nieman CC, Sanford MR, Dinis J, Martins C, Rodrigues A, Cornel AJ, et al. Asymmetric introgression between the $\mathrm{M}$ and $\mathrm{S}$ forms of the malaria vector, Anopheles gambiae, maintains divergence despite extensive hybridization. Mol Ecol. 2011;20:4983-94

19. Weetman D, Wilding CS, Steen K, Pinto J, Donnelly MJ. Gene flow-dependent genomic divergence between Anopheles gambiae $\mathrm{M}$ and $\mathrm{S}$ forms. Mol Biol Evol. 2012;29:279-91.

20. Nwakanma DC, Neafsey DE, Jawara M, Adiamoh M, Lund E, Rodrigues A, et al. Breakdown in the process of incipient speciation in Anopheles gambiae. Genetics. 2013;193:1221-31.

21. Lee $Y$, Marsden CD, Norris LC, Collier TC, Main BJ, Fofana A, et al. Spatiotemporal dynamics of gene flow and hybrid fitness between the $\mathrm{M}$ and $\mathrm{S}$ forms of the malaria mosquito, Anopheles gambiae. Proc Natl Acad Sci USA. 2013;110:19854-9.

22. Weill M, Chandre F, Brengues C, Manguin S, Akogbeto M, Pasteur N, et al. The $k d r$ mutation occurs in the Mopti form of Anopheles gambiae s.S. through introgression. Insect Mol Biol. 2000;9:451-5.

23. Gentile G, Santolamazza F, Fanello C, Petrarca V, Caccone A, della Torre A. Variation in an intron sequence of the voltage-gated sodium channel gene correlates with genetic differentiation between Anopheles gambiae s.s. molecular forms. Insect Mol Biol. 2004;13:371-7.

24. Scott JA, Brogdon WG, Collins FH. Identification of single specimens of the Anopheles gambiae complex by the polymerase chain reaction. Am J Trop Med Hyg. 1993:49:520-9.

25. Fanello C, Santolamazza F, della Torre A. Simultaneous identification of species and molecular forms of the Anopheles gambiae complex by PCR-RFLP. Med Vet Entomol. 2002;16:461-4.

26. Santolamazza F, Mancini E, Simard F, Qi Y, Tu Z, della Torre A. Insertion polymorphisms of SINE200 retrotransposons within speciation islands of Anopheles gambiae molecular forms. Malar J. 2008;7:163.

27. Martinez-Torres D, Chandre F, Williamson MS, Darriet F, Berge JB, Devonshire AL, et al. Molecular characterization of pyrethroid knockdown resistance $(\mathrm{kdr})$ in the major malaria vector Anopheles gambiae s.s. Insect Mol Biol. 1998;7:179-84.

28. Ranson H, Jensen B, Vulule JM, Wang X, Hemingway J, Collins FH. Identification of a point mutation in the voltage-gated sodium channel gene of Kenyan Anopheles gambiae associated with resistance to DDT and pyrethroids. Insect Mol Biol. 2000;9:491-7.

29. Lynd A, Ranson H, McCall PJ, Randle NP, Black WC, Walker ED, et al. A simplified high-throughput method for pyrethroid knock-down resistance (kdr) detection in Anopheles gambiae. Malar J. 2005;4:16.

30. Staden R, Beal KF, Bonfield JK. The Staden package. Methods Mol Biol. 2000;132:115-30

31. Katoh K, Kuma K, Toh H, Miyata T. MAFFT version 5: improvement in accuracy of multiple sequence alignment. Nucleic Acids Res. 2005;33:511-8. 
32. Stephens M, Smith N, Donnelly P. A new statistical method for haplotype reconstruction from population data. Am J Hum Genet. 2001;68:978-89.

33. Librado P, Rozas J. DnaSP v5: a software for comprehensive analysis of DNA polymorphism data. Bioinformatics. 2009;25:1451-2.

34. Tajima F. Statistical method for testing the neutral mutation hypothesis by DNA polymorphism. Genetics. 1989;123:585-95.

35. Fu YX, Li WH. Statistical tests of neutrality of mutations. Genetics. 1993;133:693-709.

36. Clement M, Posada D, Crandall KA. TCS: a computer program to estimate gene genealogies. Mol Ecol. 2000;9:1657-9.

37. Excoffier L, Laval G, Schneider S. Arlequin (version 3.0): an integrated software package for population genetics data analysis. Evol Bioinform Online. 2005:1:47-50.

38. Santolamazza F, Caputo B, Calzetta M, Vicente JL, Mancini E, Petrarca V, et al. Comparative analyses reveal discrepancies among results of commonly used methods for Anopheles gambiae molecular form identification. Malar J. 2011:10:215

39. Etang J, Vicente JL, Nwane P, Chouaibou M, Morlais I, Do Rosario VE, et al. Polymorphism of intron-1 in the voltage-gated sodium channel gene of Anopheles gambiae s.s. populations from Cameroon with emphasis on insecticide knockdown resistance mutations. Mol Ecol. 2009;18:3076-86.

40. Clarkson CS, Weetman D, Essandoh J, Yawson AE, Maslen G, Manske M, et al. Adaptive introgression between Anopheles sibling species eliminates a major genomic island but not reproductive isolation. Nature Comm. 2014;5:4248

41. Pinto J, Lynd A, Vicente JL, Santolamazza F, Randle NP, Moreno G, et al. Multiple origins of knockdown resistance mutations in the Afrotropical mosquito vector Anopheles gambiae. PLoS One. 2007;2:e1243.

42. Santolamazza F, Calzetta M, Etang J, Barrese E, Dia I, Caccone A, et al. Distribution of knock-down resistance mutations in Anopheles gambiae (Diptera: Culicidae) molecular forms in west and west-central Africa. Malar J. 2008;7:74.

43. Slotman MA, Tripet F, Cornel AJ, Meneses CR, Lee $Y$, Reimer $L$, et al. Evidence for subdivision within the M molecular form of Anopheles gambiae. Mol Ecol. 2007:16:639-49.

44. Pinto J, Egyir-Yawson A, Vicente J, Gomes B, Santolamazza F, Moreno M, et al. Geographic population structure of the African malaria vector Anopheles gambiae suggests a role for the forest-savannah biome transition as a barrier to gene flow. Evol Appl. 2013:6:910-24.

\section{Submit your next manuscript to BioMed Central and take full advantage of:}

- Convenient online submission

- Thorough peer review

- No space constraints or color figure charges

- Immediate publication on acceptance

- Inclusion in PubMed, CAS, Scopus and Google Scholar

- Research which is freely available for redistribution

Submit your manuscript at www.biomedcentral.com/submit 